\title{
Saturated hydrogen saline ameliorates lipopolysaccharide-induced acute lung injury by reducing excessive autophagy (Review)
}

\author{
YIMING LIU and JIN ZHANG \\ Department of Anesthesiology, Affiliated Shengjing Hospital, China Medical University, \\ Shenyang, Liaoning 110004, P.R. China
}

Received October 9, 2015; Accepted February 7, 2017

DOI: $10.3892 /$ etm.2017.4353

\begin{abstract}
The pathogenesis of acute lung injury (ALI) induced by lipopolysaccharide (LPS) involves excessive pulmonary inflammation and oxidative stress. In turn, autophagy is associated with inflammatory diseases and organ dysfunction, and studies have demonstrated that LPS treatment may trigger autophagy. Thus, excessive autophagy may stimulate the strong inflammatory response observed in the development of LPS-induced ALI. Saturated hydrogen saline may alleviate LPS-induced ALI by inhibiting autophagy, however its underlying mechanisms of action remain unknown. It has been suggested that saturated hydrogen saline may downregulate expression of nuclear factor (NF)- $\kappa \mathrm{B}$, leading to a decrease in Beclin-1 transcription and inhibition of autophagy. Inhibition of autophagy also occurs via the phosphorylation of Unc-51-like autophagy activating kinase 1 and autophagy-related protein-13 by mechanistic target of rapamycin, which in turn may be upregulated by saturated hydrogen saline. In addition, signaling pathways involving heme oxygenase-1 and p38 mitogen-activated protein kinase are associated with the alleviative effects of saturated hydrogen saline on LPS-induced autophagy. The present review focuses on potential molecular mechanisms regarding the effects of saturated hydrogen saline in the reduction of autophagy during LPS-induced ALI.
\end{abstract}

\section{Contents}

1. Introduction

2. Autophagy

3. Autophagy and the inflammatory response

4. Autophagy and LPS-induced ALI

5. Saturated hydrogen saline

Correspondence to: Professor Jin Zhang, Department of Anesthesiology, Affiliated Shengjing Hospital, China Medical University, 36 Sanhao Street, Shenyang, Liaoning 110004, P.R. China E-mail: zhangj_1@sj-hospital.org

Key words: saturated hydrogen saline, lipopolysaccharides, acute lung injury, autophagy
6. Saturated hydrogen saline and LPS-induced ALI

7. Effects of saturated hydrogen saline on autophagy during LPS-induced ALI

8. Conclusion

\section{Introduction}

Acute lung injury (ALI) and associated acute respiratory distress syndrome (ARDS) are characterized by excessive pulmonary inflammation and oxidative stress, and caused by various internal and external factors that interfere with lung function (1). Gram-negative bacterial infections are considered to be the primary cause of ALI (2). The principal active ingredient of bacterial endotoxin is lipopolysaccharide (LPS), which directly activates pulmonary inflammatory cells by interacting with cell-surface receptors, leading to high-level secretion of inflammatory mediators and prompting an inflammatory cascade that results in diffuse lung tissue damage $(3,4)$. In addition, during pathological conditions including ischemia and inflammatory ALI, large quantities of free radicals are generated that cause high levels of intracellular oxidative damage (5).

Previous studies investigating ALI-mediated cell death have principally focused on various aspects of apoptosis $(4,6)$. However, as autophagy serves as a trigger for apoptosis and necrosis (7), it is also considered to be relevant in the pathogenesis of many pulmonary diseases, including ALI. Thus, autophagy-blocking agents that prevent cell death may be developed to treat ALI (8).

Currently, there is a lack of effective therapeutics to treat ALI. Pretreatment with saturated hydrogen saline may have a protective effect against LPS-induced ALI (9); however, it remains unknown whether saturated hydrogen saline has therapeutic effects on LPS-induced autophagy in lung tissue. Signaling pathways involving nuclear factor $(\mathrm{NF})-\kappa \mathrm{B}$, Beclin-1, mechanistic target of rapamycin (mTOR), heme oxygenase-1 (HO-1) and p38 mitogen-activated protein kinase (MAPK) have been implicated in the process of autophagy, and thus may be involved in the protective effects of saturated hydrogen saline on LPS-induced ALI. Therefore, the aim of the current review was to evaluate the potential alleviative effects of saturated hydrogen saline in LPS-induced ALI, particularly regarding cell autophagy, as well as its corresponding mechanisms of action. Understanding the role of 
saturated hydrogen saline in LPS-induced ALI may determine the therapeutic effects of saturated hydrogen saline in the treatment of ALI/ ARDS.

\section{Autophagy}

Autophagy, derived from the ancient Greek word for 'self-devouring', is a tightly regulated and evolutionary conserved catabolic process, whereby damaged proteins and organelles are degraded within intracellular lysosomes (10). Autophagy may be categorized into three basic subtypes: Microautophagy, chaperone-mediated autophagy (CMA) and macroautophagy (11). Microautophagy is a nonselective process that generally involves sequestration of cytosolic proteins within invaginations of the lysosomal membrane (12). In CMA, proteins are selectively delivered to the lysosomes following recognition of an internal consensus sequence by a molecular chaperone, such as heat-shock cognate-70 (13). Macroautophagy, which will subsequently be referred to as 'autophagy', commences with the formation of a double-membrane vacuole known as an autophagosome. This acts to enclose bulk proteins and organelles within the cytosol before delivering them for lysosomal degradation, resulting in the release and recycling of amino acids and lipids (14).

All types of autophagic process consist of four main steps: i) Initiation, ii) autophagosome elongation, iii) autophagosome closure and iv) lysosomal fusion. Initiation of autophagy involves the formation of a phagophore that originates from various intracellular membrane sources. This is followed by elongation and closure of the phagopore, forming a complete autophagosome. Subsequent fusion of lysosomes with autophagosomes forms autolysosomes, in which substrates of autophagy are exposed to the hydrolytic interior of the lysosome, ultimately resulting in substrate degradation (9).

Under normal physiological conditions, low levels of autophagy occur within cells (9). However, in the absence of nutrients in the environment (resulting in starvation), the rate of autophagy increases, with greater degradation of macromolecules providing the minimum energy required to maintain cell survival. Certain toxins and pathogens are also degraded by autophagy, along with degenerative cytoplasmic components $(4,9)$. Thus, autophagy serves a key preventative role during the early stages of many infectious diseases; however its overactivation may result in excessive degradation of intracellular substances and cell death (15).

\section{Autophagy and the inflammatory response}

Stimulation of autophagy in response to nutrient deprivation ensures that minimal housekeeping functions and nutrient recycling are maintained within cells. However, autophagy-related stress, caused by dysfunction or overactivation of autophagy, typically results in intracellular degradation that exceeds the reserve capacity of cells and is associated with cell-death $(4,9)$. Excessive stimulation of autophagy is associated with pathologies that involve an altered inflammatory response, which in turn contribute to the development of various conditions, including pulmonary infections and diseases (16). In addition, autophagy itself may promote an inflammatory response (17) due to its roles in the degradation of factors associated with inflammation, namely long-lived proteins, protein aggregates and invading microbes.

The effects of autophagy on inflammatory signaling are associated with its regulation of inflammasome-dependent responses. Autophagy regulates these responses by controlling the levels of pro-inflammatory cytokine secretion for factors, including interleukin-1 $\beta$ (IL)-1 $\beta$ and IL-18. This regulation of inflammasome activity by the autophagic response is depicted in Fig. 1.

\section{Autophagy and LPS-induced ALI}

Autophagy is triggered in response to various stress stimuli, including nutrient and energy stresses, endoplasmic reticulum stress, hormone stimulation, hypoxia, redox stress and mitochondrial and DNA damage (18). Although autophagy is considered to be a key protective mechanism against stress stimuli and serves an important role in many physiological processes, excessive autophagy is associated with cell death (19).

It has been demonstrated that treatment with LPS may trigger autophagy, and that the molecular pathogenesis of ALI is associated with LPS-induced autophagic cell death (20). Additionally, increased levels of autophagosomes and microtubule-associated protein 1A/ 1B-light chain 3 (LC3) expression have been observed in lung cells following LPS treatment, along with the increased expression of other autophagy-related proteins associated with the formation and maturation of autophagosomes and autolysosomes, namely autophagy-related protein (Atg) 4, Atg 7 and the Atg5-Atg12-Atg16 complex. Furthermore, it has been demonstrated that genetic depletion of the primary autophagy mediators LC3 and Beclin-1 suppresses LPS-induced lung cell death, suggesting that autophagic cell death may be the basic mechanism underlying the molecular pathogenesis of LPS-induced ALI $(9,21)$.

\section{Saturated hydrogen saline}

Hydrogen is the lightest and most abundant chemical element, and molecular hydrogen is an odorless, colorless, tasteless, nonmetallic and highly flammable diatomic gas (9). Hydrogen is considered to be a novel anti-oxidant that may be capable of quenching free radicals. Studies performed in vitro and in vivo have indicated that hydrogen may be effective at treating brain (22), liver (5), heart (23) and intestinal (24) ischemia reperfusion injuries. The efficacy of hydrogen as an anti-inflammatory therapy has also been demonstrated in the treatment of acute pancreatitis (25), enteritis (26) and hepatitis (3). The radical-quenching properties of hydrogen are due to its ability to spread rapidly and form water upon contact with hydroxyl radicals. Physiologically, inhalation of low concentrations of hydrogen is considered to be relatively safe, though in clinical practice, the use of hydrogen is limited to antimicrobial therapies (27). Previous studies have demonstrated that saturated hydrogen saline (saline saturated in $\mathrm{H}_{2}$ ), is a safe and easily administrated compound, has antioxidant, anti-inflammatory and anti-apoptotic effects, and provides protection against numerous diseases, including sepsis, stroke, atherosclerosis, multiple organ dysfunction syndrome and nigrostriatal degeneration $(22,23)$. It has been determined that saturated hydrogen 


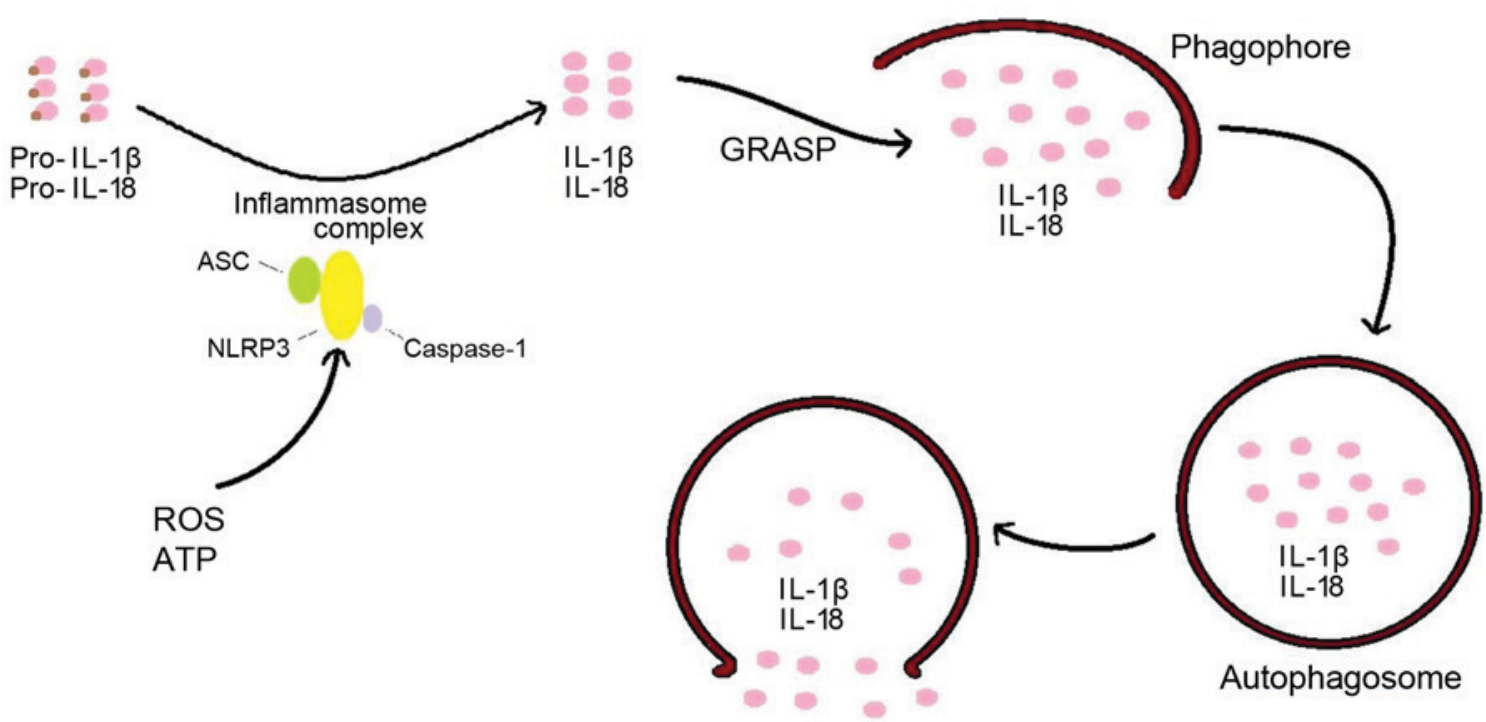

Figure 1. Involvement of autophagy in the secretion of IL-1 $\beta$ and IL-18. ROS and ATP trigger the activation of the sensor molecule NLRP3. The activation of NLRP3 stimulates assembly of inflammasome complexes through the recruitment of the adaptor protein ASC and pro-caspase-1, leading to the cleavage of the caspase proform. Activated caspase- 1 subsequently cleaves the pro-inflammatory cytokine precursors pro-IL-1 $\beta$ and pro-IL- 18 into their biologically active forms (IL-1 $\beta$ and IL-18). Active IL-1 $\beta$ and IL-18 are transported into autophagic vesicles via GRASP proteins and secreted outside of the cell through autophagic vesicles. IL, interleukin; ROS, reactive oxygen species; ATP, adenosine triphosphate; NLRP3, NLR Family Pyrin Domain Containing 3; ASC, Apoptosis-associated speck-like protein containing a caspase recruitment domain; GRASP, golgi reassembly and stacking protein.

saline selectively removes cellular hydroxyl radicals and exerts antioxidant effects, thus protecting cells and tissues from oxidative damage (28). In addition, Xu and Zhang (4) demonstrated that saturated hydrogen saline reduced liver dysfunction induced by LPS, while Gu et al (29) documented that saturated hydrogen saline may alleviate LPS-induced acute kidney injury. These data suggest that saturated hydrogen saline may have similar protective effects in LPS-induced ALI.

\section{Saturated hydrogen saline and LPS-induced ALI}

Saturated hydrogen saline significantly prolongs the median survival time and prevents pulmonary tissue damage in rats following LPS treatment. Specifically, it has been demonstrated that LPS stimulation led to alveolar wall thickening, edema and hemorrhage, reduced alveolar space, substantial inflammatory cell infiltration and the presence of autophagosomes in type II alveolaries. All of these histopathological changes were attenuated by treatment with hydrogen saline (30). Primary characteristics associated with LPS-induced ALI are excessive pulmonary inflammation and increased vascular permeability, and in some cases, pulmonary edema. This results in elevated levels of total protein within the bronchoalveolar lavage fluid (BALF) (21). It has been documented that saturated hydrogen saline may reverse increases in lung wet/ dry ratio and total protein concentration in the BALF following LPS stimulation, suggesting that saturated hydrogen saline may alleviate LPS-induced pulmonary edema (21).

\section{Effects of saturated hydrogen saline on autophagy during LPS-induced ALI}

It has been demonstrated that the secretion of pulmonary inflammatory cytokines, namely tumor necrosis factor
(TNF)- $\alpha$, IL-1 and IL-6, increases following LPS treatment, reflecting the high-level secretion of inflammatory factors that occurs during ALI (9). However, levels of cytokine secretion decreased markedly following treatment with saturated hydrogen saline, indicating that saturated hydrogen saline may inhibit an LPS-induced inflammatory response. Interestingly, physical signs of autophagy were more pronounced following LPS treatment and predominantly observed in the cytoplasm of type II alveolar epithelial cells (31). In addition, the expression of autophagy-related proteins, including LC3, Atg4, Atg7, the Atg5-Atg12-Atg16 complex and Beclin-1, increased in lung tissue following LPS treatment, suggesting that the entire process of autophagy was activated following LPS-induced inflammation (9). As saturated hydrogen saline reduces the expression of autophagy-related proteins and the number of autophagosomes within type II alveolaries (9), it may function as a protective agent against LPS-induced ALI by reducing excessive autophagy. Hydrogen saline is considered to alleviate autophagy by five potential mechanisms, as discussed in sections 7.1 to 7.5 .

$N F-\kappa B$ and Beclin-1. Among the core Atg proteins, Beclin-1, as the orthologue of Atg6, contains a coiled-coil motif that binds to the Class III phosphatidylinositol-3-kinase vacuolar protein sorting (Vps) -34, which stimulates the generation of the key autophagosome component phosphatidylinositol-3-phosphate, leading to autophagosome formation (32). Three distinct Beclin-1-Vps34 complexes have been identified that include Beclin-1, Vps34 and Vps15 as common components: One containing Beclin-1, Vps34, Vps15 and Atg14L; one containing Beclin-1, Vps34, Vps15 and ultraviolet irradiation resistance-associated gene (UVRAG); and one containing Beclin-1, Vps34, Vps15, UVRAG and Rubicon (33). Notably, the complex containing Atg14 L functions in autophagosome 
formation while others function in autophagosome and endosome maturation (33). Thus, Beclin-1 first acts as a platform in combination with other proteins to recruit activators and inhibitors of autophagy, and subsequently acts as a regulator of autophagosome formation.

It has been demonstrated that $\mathrm{NF}-\kappa \mathrm{B}$ is an important checkpoint required for the activation of autophagy. Copetti et al (34) determined that the NF- $\mathrm{B}$ family member p65 (also known as v-rel reticuloendotheliosis viral Oncogene homolog A) upregulated the expression of Beclin-1 at the mRNA and protein in different cellular systems. Furthermore, decreases in Beclin-1 transcription were observed following the inhibition of the p65 signaling pathway.

As NF- $\kappa$ B and Beclin-1 serve critical roles in the activation and progression of cell autophagy $(35,36)$, both molecules may be involved in saturated hydrogen saline-mediated lung protection. Indeed, elevated nuclear expression of $\mathrm{NF}-\kappa \mathrm{B}$ and cyto plasmic expression of Beclin-1 has been observed in lung tissues following LPS treatment, while saturated hydrogen saline treatment decreased the expression of NF- $\mathrm{BB}$ (37) and Beclin-1, thus inhibiting autophagy (38). Results from western blot analysis confirmed these findings (21). The potential involvement of $\mathrm{NF}-\kappa \mathrm{B}$ and Beclin-1 in saturated hydrogen saline-mediated lung protection is presented in Fig. 2.

Mammalian target of rapamycin. Different signaling mechanisms are involved in modulating autophagy within mammalian cells (39). The best-characterized pathways are those that modulate autophagy in response to nutritional changes and the evolutionarily conserved serine-threonine kinase mTOR, which serves as a sensor of environmental and cellular nutrition, as well as cellular energy status. In higher eukaryotes, mTOR is found in at least two distinct multiprotein complexes, referred to as mTOR complex (mTORC) 1 and mTORC $2(40,41)$. mTORC1 is associated with an additional complex of proteins consisting of unc-51-like autophagy activating kinase 1 (ULK1; a mammalian analog of the yeast master regulator of autophagy) Atg1 (the mammalian analog of Atg13) and the scaffold protein focal adhesion kinase-family interacting protein of $200 \mathrm{kDa}$ (FIP200) (42). mTOR serves a key regulatory role in the regulation of autophagy and inhibits autophagy by phosphorylating ULK1 and Atg13 $(43,44)$. However, during cell starvation, mTORC1 dissociates from the Atg13-FIP200-ULK1 complex, leading to dephosphorylation of ULK1 and Atg13, and activation of ULK1, which in turn phosphorylates Atg13 and FIP200 (45). These events activate autophagy, whereby a pre-autophagosomal membrane forms as an autophagosome precursor.

It has been demonstrated that small doses of reactive oxygen species (ROS) activate mTOR signaling, while high levels of ROS induced by LPS inhibit the mTOR signaling pathway (21). In addition, hypoxia results in the accumulation of free radicals that inhibit mTOR (46). Previous studies have documented that saturated hydrogen saline increases the expression of mTOR protein within skeletal muscle $(47,48)$. Experimental results have also indicated that saturated hydrogen saline may remove excess free radicals, decrease the concentration of free radicals in lung tissue, promote mTOR expression and prevent excessive autophagy (Fig. 3) (9,21).
Toll-like receptors. Toll-like receptors (TLRs) are transmembrane pattern recognition receptors. As components of the innate immune system, they are key elements in the recognition of viral and bacterial factors (49). The association between autophagy, inflammation and the activation of TLR signaling pathways has recently been documented (50). Detection of microbes by TLRs stimulates autophagy and an inflammatory response. Furthermore, it has been observed that TLR4 specifically can be activated to initiate an immune response by antigen-presenting naive $\mathrm{T}$ cells following LPS sensitization (51).

In macrophages, LPS may induce autophagy through TLR4. This involves an adaptor complex comprised of TLR-domain-containing adapter-inducing interferon- $\beta$ and receptor-interacting serine/ threonine-protein kinase 1 , and the p38 MAPK signaling pathway (50). These data suggest that TLR signaling pathways are potential mediators of LPS-induced autophagy. Thus, the ameliorative effects of saturated hydrogen saline on autophagy and inflammation may occur, at least partly, through TLR signaling pathways.

HO-1 signaling. HO is involved in the degradation of heme, which produces iron, bilirubin and carbon monoxide. Three forms of $\mathrm{HO}, \mathrm{HO}-1, \mathrm{HO}-2$ and $\mathrm{HO}-3$, have been identified (52). HO-1, a major heat shock/stress response protein, is an endogenous cell-protective protein that assists in the prevention of oxidative stress, inflammation and excessive cell autophagy $(53,54)$. In addition, HO-1 modulates the release of inflammatory cytokines in LPS-stimulated macrophages, sepsis and intestinal inflammation (55).

It has been demonstrated that induction of HO-1 expression may reduce the sensitivity of animal models to lethal doses of LPS, thereby improving survival rates of animals treated with LPS (52). Furthermore, the HO-1/ monoxide pathway serves a key role in protecting tissues against damage caused by LPS. HO-1 has been associated with both the cytoprotective and cytotoxic functions of autophagy induction. Inducible HO-1 overexpressing cells significantly inhibit ROS generation and delay the onset of autophagy (52). It has been demonstrated that HO-1 inhibits the cytotoxic effects of autophagy in lung epithelial cells in response to LPS, by downregulating autophagy-related signaling (56). Furthermore, studies have also indicated that saturated hydrogen saline may increase HO-1 expression $(52,57)$. The current review of experimental results indicates that saturated hydrogen saline ameliorates excessive activation of autophagy and ALI under high LPS conditions. The underlying mechanism regarding the protective effects of saturated hydrogen saline against LPS-induced lung damage may involve upregulation of $\mathrm{HO}-1$ and subsequent inhibition of excessive autophagy.

p38 MAPK signaling. MAPK cascades are involved in a variety of cellular processes. However, MAPK signaling may also be activated by oxidative stress and contribute to tissue damage, as observed in pneumocyte injury (9). MAPK pathways are mediated by ERK, JNK and p38 protein kinases (58), all of which are activated by pro-inflammatory cytokines including TNF- $\alpha$ and IL-6 (59). In a cell model of oxidative damage using human trophoblast-like cells, it was observed that treatment 


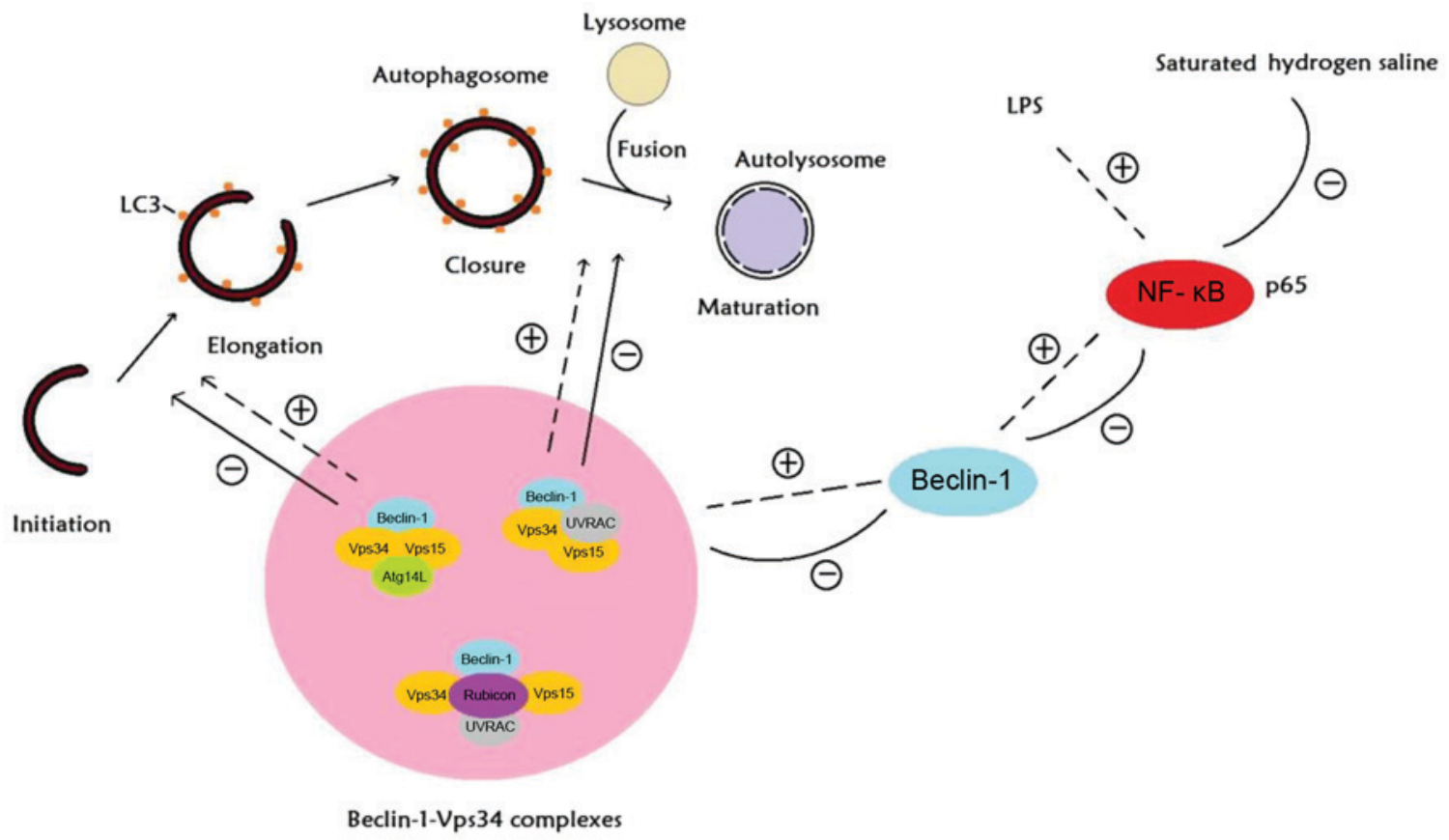

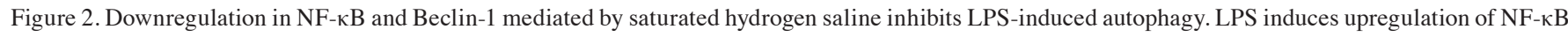
and Beclin-1 mRNA and protein, promoting the formation of Beclin-1-Vps34 complexes and autophagy. Saturated hydrogen saline reduces nuclear expression of NF- $\mathrm{kB}$ and cytoplasmic expression of Beclin-1, leading to a reduction in Beclin-1-Vps34 complexes. A lack of Beclin-1-Vps34-Atg14L complexes hinders

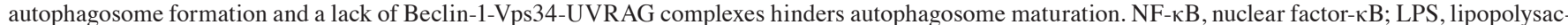
charide; Vps, Vacuolar protein sorting; Atg, autophagy-related protein; UVRAG, ultraviolet radiation resistance-associated gene.



Autophagy

Figure 3. Upregulation in mTOR mediated by saturated hydrogen saline inhibits LPS-induced autophagy. High levels of ROS generation induced by LPS inhibits mTOR, leading to a decrease in the phosphorylation of Atg13 and ULK1. Unphosphorylated ULK1 exhibits kinase activity, triggering phosphorylation of FIP200 and Atg13 and activating the Atg13-FIP200-ULK1 complex. Translocation of the complex to the pre-autophagosome promotes autophagy. Saturated hydrogen saline promotes the expression of mTOR, leading to dephosphorylation of Atg13 and ULK1 and thus inhibiting autophagy. mTOR, mechanistic target of rapamycin; LPS, lipopolysaccharide; ROS, reactive oxygen species; Atg, autophagy-related protein; ULK1, unc-51 like autophagy activating kinase 1; FIP200, focal adhesion kinase-family interacting protein of $200 \mathrm{kDa}$.

with saturated hydrogen saline efficiently regulated the activation of ERK, JNK and p38 (60), suggesting an involvement of MAPK cascades in saturated hydrogen saline-mediated lung protection. However, an association between MAPK signaling and oxidative stress or inflammatory responses has yet to be identified. 
Studies conducted in vitro have indicated an involvement of p38 MAPK in the expression of multiple genes during conditions of stress, particularly in cytokine synthesis signal transduction during LPS-induced ALI $(9,61)$. The p38 MAPK pathway has also been associated with a variety of pathways involved in apoptosis, cell cycle arrest and autophagic death (60), suggesting that the p38 MAPK pathway may be involved in the effects of saturated hydrogen saline on autophagy during ALI. Experimental results have determined that the specific MAPK inhibitor SB203580 is able to inhibit the expression of p38 and thereby reduce autophagy in LPS-sensitized lung cells (61). Furthermore, levels of p38 and LC3 following LPS exposure were all significantly reduced following saturated hydrogen saline treatment, indicating that p38 may be involved in the mechanism by which saturated hydrogen saline prevents excessive autophagy in lung cells (62).

\section{Conclusion}

The pathophysiology of ALI/ ARDS is complex and involves excessive levels of cell autophagy. In LPS-induced ALI, saturated hydrogen saline may reduce levels of inflammation not only by inhibiting apoptosis, but also by inhibiting excessive autophagy. In particular, saturated hydrogen saline may inhibit the expression of autophagy-related genes, thus reducing autophagy in injured lung cells and improving the survival rate of lung tissue. Saturated hydrogen saline may downregulate $\mathrm{NF}-\kappa \mathrm{B}$ and Beclin-1, and upregulate mTOR and LC3B, thus inhibiting excessive autophagy. Furthermore, saturated hydrogen saline may stimulate $\mathrm{HO}-1$ signaling and inhibit p38 MAPK signaling. However, the specific mechanism of this remains unknown. Indeed, there may be an association between these different pathways, which warrants further investigation.

There is currently a lack of effective therapeutics available to treat ALI. Saturated hydrogen saline presents a novel therapeutic approach for the treatment of ALI, where it may aid in protecting against lung injury. However, the underlying mechanisms regarding the effects of saturated hydrogen saline are complex and further studies are required to elucidate its molecular involvement in the pathways associated with LPS-induced ALI.

\section{Acknowledgements}

The present study was supported by the Shenyang Municipal Science and Technology commission, Shenyang, China (grant nos. F10-205-1-67 and F14-231-60).

\section{References}

1. Butt Y,Kurdowska A and Allen TC: Acute lung injury: A clinical and molecular review. Arch Pathol Lab Med 140: 345-350, 2016.

2. Chew KC, Ang ET, Tai YK, Tsang F, Lo SQ, Ong E, Ong WY, Shen HM, Lim KL, Dawson VL, et al: Enhanced autophagy from chronic toxicity of iron and mutant A53T $\alpha$-synuclein: Implication for neuronal cell death in Parkinson disease. J Biol Chem 286: 33380-33389, 2011.

3. Tamura H, Reich $\mathbf{J}$ and Nagaoka I: Bacterial endotoxin assays relevant to host defense peptides. Junten Med J 62: 132-140, 2016.

4. Xu XF and Zhang J: Saturated hydrogen saline attenuates endotoxin-induced acute liver dysfunction in rats. Physiol Res 62: 395-403, 2013.
5. Lingappan K, Jiang W, Wang L, Wang G, Couroucli XI, Shivanna B, Welty SE, Barrios R, Khan MF, Nebert DW, et al: Mice deficient in the gene for cytochrome P450 (CYP) 1A1 are more susceptible than wild type to hyperoxic lung injury: Evidence for protective role of CYP1A1 against oxidative stress. Toxicol Sci 141: 68-77, 2014.

6. Chopra M, Reuben JS and Sharma AC: ALI: Apoptosis and signaling mechanisms. Exp Biol Med (Maywood) 234: 361-371, 2009.

7. Dong Y, Undyala VV, Gottlieb RA, Mentzer RM Jr and Przyklenk K: Autophagy: Definition, molecular machinery, and potential role in myocardial ischemia-reperfusion injury. J Cardiovasc Pharmacol Ther 15: 220-230, 2010.

8. Liu Q, Zhou D, Lin P, Gao XL, Pan L and Jin FG: Participation of autophagy in acute lung injury induced by seawater. Exp Lung Res 39: 441-452, 2013.

9. Zhang Y, Liu Y and Zhang J: Saturated hydrogen saline attenuates endotoxin-induced lung dysfunction. J Surg Res 198: 41-49, 2015.

10. Czarny P, Pawlowska E, Bialkowska-Warzecha J, Kaarniranta K and Blasiak J: Autophagy in DNA damage response. Int J Mol Sci 16: 2641-2662, 2015.

11. Al-Ejeh F, Kumar R, Wiegmans A, Lakhani SR, Brown MP and Khanna KK: Harnessing the complexity of DNA-damage response pathways to improve cancer treatment outcomes. Oncogene 29: 6085-6098, 2010.

12. Sahu R, Kaushik S, Clement CC, Cannizzo ES, Scharf B, Follenzi A, Potolicchio I, Nieves E, Cuervo AM and Santambrogio L: Microautophagy of cytosolic proteins by late endosomes. Dev Cell 20: 131-139, 2011.

13. Klionsky DJ, Abdalla FC, Abeliovich H, Abraham RT, Acevedo-Arozena A, Adeli K, Agholme L, Agnello M, Agostinis P, Aguirre-Ghiso JA, et al: Guidelines for the use and interpretation of assays for monitoring autophagy. Autophagy 8: 445-544, 2012.

14. Filomeni G, De Zio D and Cecconi F: Oxidative stress and autophagy: The clash between damage and metabolic needs. Cell Death Differ 22: 377-388, 2015.

15. Mizumura K, Cloonan SM, Haspel JA and Choi AM: The emerging importance of autophagy in pulmonary diseases autophagy in pulmonary disease. Chest 142: 1289-1299, 2012.

16. Liu Q, Zhou D, Sun L, Ling L, Wu CG, Lin P and Han SP: Bone marrow mesenchymal stem cells ameliorates seawater-exposure-induced acute lung injury by inhibiting autophagy in lung tissue. Patholog Res Int 2014: 104962, 2014.

17. Karlsson S, Pettilä V, Tenhunen J, Laru-Sompa R, Hynninen M and Ruokonen E: HMGB1 as a predictor of organ dysfunction and outcome in patients with severe sepsis. Intensive Care Med 34: 1046-1053, 2008.

18. Wei H, Wang C, Croce CM and Guan JL: p62/ SQSTM1 synergizes with autophagy for tumor growth in vivo. Genes Dev 28: 1204-1216, 2014.

19. Rodriguez-Rochaa H, Garcia-Garciaa A, Panayiotidisb MI and Francoa R: DNA damage and autophagy. Mutat Res 711: 158-166, 2011.

20. Mizumura K, Cloonan SM, Haspel JA and Choi AM: The emerging importance of autophagy in pulmonary diseasesautophagy in pulmonary disease. Chest 142: 1289-1299, 2012.

21. Gao Y, Wang N, Liu L, Liu Y and Zhang J: Relationship between mammalian target of rapamycin and autophagy in lipopolysaccharide-induced lung injury. J Surg Res 201: 356-363, 2016.

22. Cai J, Kang Z, Liu K, Liu W, Li R, Zhang JH, Luo X and Sun X: Neuroprotective effects of hydrogen saline in neonatal hypoxia-ischemia rat model. Brain Res 1256: 129-137, 2009.

23. Sun Q, Kang Z, Cai J, Liu W, Liu Y, Zhang JH, Denoble PJ, Tao $\mathrm{H}$ and Sun X: Hydrogen-rich saline protects myocardium against ischemia/ reperfusion injury in rats. Exp Biol Med (Maywood) 234: 1212-1219, 2009.

24. Zheng X, Mao Y, Cai J, Li Y, Liu W, Sun P, Zhang JH, Sun X and Yuan H: Hydrogen-rich saline protects against intestinal ischemia/ reperfusion injury in rats. Free Radic Res 43: 478-484, 2009.

25. Chen H, Sun YP, Li Y, Liu WW, Xiang HG, Fan LY, Sun Q, $\mathrm{Xu}$ XY, Cai JM, Ruan CP, et al: Hydrogen-rich saline ameliorates the severity of 1-arginine-induced acute pancreatitis in rats. Biochem Biophys Res Commun 393: 308-313, 2010.

26. Kajiya M, Silva MJ, Sato K, Ouhara K and Kawai T: Hydrogen mediates suppression of colon inflammation induced by dextran sodium sulfate. Biochem Biophys Res Commun 386: 11-15, 2009. 
27. Yanong RPE: Use of Hydrogen Peroxide in Finfish Aquaculture1 Institute of Food and Agricultural Sciences, University of Florida, Gainesville, FL, 2008.

28. Yuan H, Perry CN, Huang C, Iwai-Kanai E, Carreira RS, Glembotski CC and Gottlieb RA: LPS-induced autophagy is mediated by oxidative signaling in cardiomyocytes and is associated with cytoprotection. Am J Physiol Heart Circ Physiol 296: H470-H479, 2009.

29. Gu H, Yang M, Zhao X, Zhao B, Sun X and Gao X: Pretreatment with hydrogen-rich saline reduces the damage caused by glycerol-induced rhabdomyolysis and acute kidney injury in rats. J Surg Res 188: 243-249, 2014.

30. Fine KL, Metcalfe MG, White E, Virji M, Karls RK and Quinn FD: Involvement of the autophagy pathway in trafficking of Mycobacterium tuberculosis bacilli through cultured human type II epithelial cells. Cell Microbiol 14: 1402-1414, 2012.

31. Pan H, Zhang Y, Luo Z, Li P, Liu L, Wang C, Wang H, Li H and $\mathrm{Ma}$ Y: Autophagy mediates avian influenza H5N1 pseudotyped particle-induced lung inflammation through NF- $\mathrm{KB}$ and p38 MAPK signaling pathways. Am J Physiol Lung Cell Mol Physiol 306: L183-L195, 2014.

32. Itakura $\mathrm{E}$, Kishi $\mathrm{C}$, Inoue $\mathrm{K}$ and Mizushima N: Beclin 1 forms two distinct phosphatidylinositol 3-kinase complexes with mammalian Atg14 and UVRAG. Mol Biol Cell 19: 5360-5372, 2008.

33. Wirth M, Joachim J and Tooze SA: Autophagosome formation-the role of ULK1 and Beclin1-PI3KC3 complexes in setting the stage (C). Semin Cancer Biol 23: 301-309, 2013.

34. Copetti T, Bertoli C, Dalla E, Demarchi F and Schneider C: p65/ RelA modulates BECN1 transcription and autophagy. Mol Cell Biol 29: 2594-2608, 2009.

35. Ishaq M, Khan MA, Sharma K, Sharma G, Dutta RK and Majumdar S: Gambogic acid induced oxidative stress dependent caspase activation regulates both apoptosis and autophagy by targeting various key molecules (NF- $\kappa \mathrm{B}$, Beclin-1, p62 and NBR1) in human bladder cancer cells. Biochim Biophys Acta 1840: 3374-3384, 2014.

36. Haar L, Ren X, Liu Y, Koch SE, Goines J, Tranter M, Engevik MA, Nieman M, Rubinstein J and Jones WK: Acute consumption of a high-fat diet prior to ischemia-reperfusion results in cardioprotection through NF- $\kappa \mathrm{B}$-dependent regulation of autophagic pathways. Am J Physiol Heart Circ Physiol 307: H1705-H1713, 2014.

37. Hu W, Wang G, Li P, Wang Y, Si CL, He J, Long W, Bai Y, Feng $Z$ and Wang X: Neuroprotective effects of macranthoin $G$ from Eucommia ulmoides against hydrogen peroxide-induced apoptosis in PC12 cells via inhibiting NF- $\kappa \mathrm{B}$ activation. Chem Biol Interact 224: 108-116, 2014.

38. Seo G, Kim SK, Byun YJ, Oh E, Jeong SW, Chae GT and Lee SB Hydrogen peroxide induces Beclin 1-independent autophagic cell death by suppressing the mTOR pathway via promoting the ubiquitination and degradation of Rheb in GSH-depleted RAW 264.7 cells. Free Radic Res 45: 389-399, 2011.

39. Mehrpour M, Esclatine A, Beau I and Codogno P: Overview of macroautophagy regulation in mammalian cells. Cell Res 20 748-762, 2010.

40. Zoncu R, Efeyan A and Sabatini DM: mTOR: From growth signal integration to cancer, diabetes and ageing. Nat Rev Mol Cell Biol 12: 21-35, 2010

41. Hsu PP, Kang SA, Rameseder J, Zhang Y, Ottina KA, Lim D, Peterson TR, Choi Y, Gray NS, Yaffe MB, et al: The mTOR-regulated phosphoproteome reveals a mechanism of mTORC1-mediated inhibition of growth factor signaling. Science 332: 1317-1322, 2011

42. Mizushima N: The role of the Atg1/ ULK1 complex in autophagy regulation. Curr Opin Cell Biol 22: 132-139, 2010.

43. Wei H and Guan JL: Pro-tumorigenic function of autophagy in mammary oncogenesis. Autophagy 8: 129-131, 2012.

44. Dunlop EA and Tee AR: mTOR and autophagy: A dynamic relationship governed by nutrients and energy (C). Seminars in cell \& developmental biology. Academic Press 36: 121-129, 2014.
45. Roberts DJ and Miyamoto S: Hexokinase II integrates energy metabolism and cellular protection: Akting on mitochondria and TORCing to autophagy. Cell Death Differ 22: 364, 2015.

46. Hu Y, Liu J, Wu YF, Lou J, Mao YY, Shen HH and Chen ZH: mTOR and autophagy in regulation of ALI: A review and perspective. Microbes Infect 16: 727-734, 2014.

47. Roschel H, Ugrinowistch C, Barroso R, Batista MA, Souza EO, Aoki MS, Siqueira-Filho MA, Zanuto R, Carvalho CR, Neves M, et al: Effect of eccentric exercise velocity on akt/ mtor/ p70(s6k) signaling in human skeletal muscle. Appl Physiol Nutr Metab 36: 283-290, 2011.

48. Proceedings of the Tenth International Society of Sports Nutrition (ISSN) conference and expo. J Int Soc Sports Nutr 10 (Suppl 1): P1-P28, 2013.

49. Lavieri R, Piccioli P, Carta S, Delfino L, Castellani P and Rubartelli A: TLR costimulation causes oxidative stress with unbalance of proinflammatory and anti-inflammatory cytokine production. J Immunol 192: 5373-5381, 2014

50. Pla A,Pascual M, Renau-Piqueras J and Guerri C: TLR4 mediates the impairment of ubiquitin-proteasome and autophagy-lysosome pathways induced by ethanol treatment in brain. Cell Death Dis 5: e1066, 2014

51. Muto J, Morioka Y, Yamasaki K, Kim M, Garcia A, Carlin AF, Varki A and Gallo RL: Hyaluronan digestion controls DC migration from the skin. J Clin Invest 124: 1309-1319, 2014

52. Liu G, Song D, Jiang Y and Ge Y: Effect of hydrogen-saline on lung injury and heme oxygenase-1 expression in the lung tissue of acute paraquat-intoxicated mice. Zhonghua Lao Dong Wei Sheng Zhi Ye Bing Za Zhi 33: 337-341, 2015 (In Chinese).

53. Dare AJ, Phillips AR, Hickey AJ, Mittal A, Loveday B, Thompson $\mathrm{N}$ and Windsor JA: A systematic review of experimental treatments for mitochondrial dysfunction in sepsis and multiple organ dysfunction syndrome. Free Radic Biol Med 47: $1517-1525,2009$

54. Gozzelino R, Jeney V and Soares MP: Mechanisms of cell protection by heme oxygenase-1. Annu Rev Pharmacol Toxicol 50: 323-354, 2010.

55. Li Q, Zhu Y, Jiang H, Jiang H, Xu H and Sun Y: Heme oxygenase-1 mediates the anti-inflammatory effect of isoflurane preconditioning in LPS-stimulated macro-phages. Acta Pharmacol Sin 30: 228-234, 2009

56. Kim HP, Wang X, Chen ZH, Lee SJ, Huang MH, Wang Y, Ryter SW and Choi AM: Autophagic proteins regulate cigarette smoke-induced apoptosis: Protective role of heme oxygenase-1. Autophagy 4: 887-895, 2008.

57. Huang CS, Kawamura T, Toyoda Y and Nakao A: Recent advances in hydrogen research as a therapeutic medical gas. Free Radic Res 44: 971-982, 2010

58. Amsterdam A, Shpigner L, Raanan C, Schreiber L, Melzer E and Seger R: Dynamic distribution of ERK, p38 and JNK during the development of pancreatic ductal adenocarcinoma. Acta Histochem 116: 1434-1442, 2014.

59. Wang Y, Tu Q, Yan W, Xiao D, Zeng Z, Ouyang Y, Huang L, Cai J, Zeng X, Chen YJ and Liu A: CXC195 suppresses proliferation and inflammatory response in LPS-induced human hepatocellular carcinoma cells via regulating TLR4-MyD88-TAK1-mediated NF- $\kappa$ B and MAPK pathway. Biochem Biophys Res Commun 456: 373-379, 2015.

60. Tang C, Liang J, Qian J, Jin L, Du M, Li M and Li D: Opposing role of JNK-p38 kinase and ERK1/ 2 in hydrogen peroxide-induced oxidative damage of human trophoblast-like JEG-3 cells. Int J Clin Exp Pathol 7: 959-968, 2014.

61. Muniyappa H and Das K C: Activation of c-Jun N-Terminal Kinase (JNK) by widely used specific p38 MAPK inhibitor SB202190 and SB203580: A MLK-3-MKK7-dependent mechanism. Cell Signal 20: 675-683, 2008

62. Liu Q, Shen WF, Sun HY, Fan DF, Nakao A, Cai JM, Yan G, Zhou WP, Shen RX, Yang JM and Sun XJ: Hydrogen-rich saline protects against liver injury in rats with obstructive jaundice. Liver Int 30: 958-968, 2010 